\title{
Non-Aqueous Route for the Synthesis of Copper Organosol from Copper Stearate: An Effective Catalyst for the Synthesis of Octylphenyl Ether
}

\author{
Sudipa Panigrahi, ${ }^{\bullet}$ Subrata Kundu, ${ }^{\#}$ Soumen Basu, ${ }^{\bullet}$ Snigdhamayee Praharaj, ${ }^{\bullet}$ \\ Subhra Jana, ${ }^{\bullet}$ Surojit Pande, ${ }^{\bullet}$ Sujit Kumar Ghosh, ${ }^{\bullet}$ Anjali Pal,,$\stackrel{\text {, }}{ }$ and \\ Tarasankar Pal ${ }^{\bullet *}$ \\ - Department of Chemistry, Indian Institute of Technology, Kharagpur-721302, India \\ E-mail: tpal@chem.iitkgp.ernet.in \\ ${ }^{\#}$ Department of Chemical Engineering, University of Nebraska, Lincoln, USA

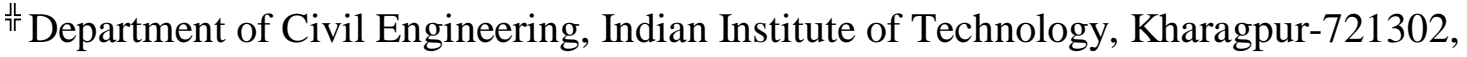

\begin{abstract}
Good quality highly stable copper organosol has been synthesized in a non hydrolytic approach from the single phase reduction of the precursor $\mathrm{Cu}$ (II) stearate by sodium borohydride in toluene under nitrogen gas atmosphere. Surface modification of the synthesized copper organosol with an amino acid, L-cysteine and an alkanethiol (1dodecanethiol, DDT) is accomplished by the thiolate bond formation between the ligands and the nanoparticle surface. Cysteine molecule binds the copper surface via a thiolate bond and amine linkage but not by the electrostatic interaction with the carboxylate group due to solvent polarity and dielectric medium of the solvent. The synthesized copper particles have been characterized by the optical spectroscopy (UV/VIS), electron microscopy (TEM), XPS and XRD. FTIR analysis was performed to confirm the surface functionalization of the copper particles either by the amino acid, L-cysteine or DDT. The synthesized particles are stable for a couple of months without alteration of the spectral profile. After that the copper particles leads to aggregation via hydrogen bond formation between the amino acid molecules located on the neighboring copper particles. It was found that only DDT (without the need of any reducing agent) could reduce copper stearate to $\mathrm{Cu}(0)$ but the synthesized particles remain stable for a week only. It was also observed that the chain length of the alkanethiols does not affect the particle morphology remarkably but has a bearing on the stability of the evolved particles. Furthermore, the synthesized copper particles have been found to serve as an effective catalyst for the synthesis of octlyphenyl ether under nitrogen gas atmosphere.
\end{abstract}




\section{Introduction}

Copper is one of the most widely used materials in the world. It has great significance in all industries, particularly in the electrical and catalytic one. ${ }^{1}$ Metallic copper has shown catalytic activity for the formation of aldehydes from methanol and ethanol. $^{2,3}$ Micro-copper particles have been employed on a polyaniline films as the chemically modified electrodes for the detection of amino acids and polyhydric molecules. ${ }^{4}$ Gedanken et al. have shown that copper particles could be employed as an effective catalyst for the Ullmann type reactions. ${ }^{5}$ Copper particles, for example, are known to enhance the catalytic activity and selectivity of $\mathrm{ZnO}$ in hydration and dehydration reactions e.g., methanol synthesis, and play an important role in solid-oxide fuel cells. ${ }^{6}$ Manbeck and coworkers have used copper clusters as a catalyst for carbonhetero atom bond formation. ${ }^{7}$ Wang et $a l .{ }^{8}$ have synthesized copper thin films on indium tin oxide and used this matrix as a catalyst for the reduction of nitrite and nitric oxides.

Since most of the reactions are carried out in organic phase, it is desirable to fabricate such a catalyst in organic solvent system. Moreover, the high surface energy of the nanoparticles ${ }^{9}$ and consequently the tendency for agglomeration hamper their preparation in high concentration in an aqueous solution. The use of organic solvents, instead of an aqueous one, might be a promising solution to this problem, where the particles gain steric stabilization due to the presence of giant hydrophobic stabilizing ligands. ${ }^{10}$ A variety of wet chemical methods have been developed thus far for the nanocrystal synthesis. The main issues are to have control over particle size and size distribution, surface passivation and core crystallinity of metal nanocrystal. ${ }^{11-13}$ In a good solvent, the ligands extend from the nanocrystal surface and provide steric stabilization, which typically limits size of the generated particles in the nanometer range and prevents unwanted agglomeration. To prevent nanosized particles from aggregation capping ligands have to anchor to the particle surface. Copper nanoparticles have been synthesized and characterized by different methods. ${ }^{14-17}$ Pioneering work by Pileni ${ }^{18}$ for the synthesis of copper nanoparticles in AOT reverse micelle system has set for the methods of synthesis of copper particles via reduction of $\mathrm{Cu}^{2+}$ ion. Recently Schmittel et al. reported the synthesis of copper nanoparticles in organic solvent by supramolecular approach $^{19}$ and harsh synthetic condition. Organometallic precursors have also been used 
for the synthesis of copper organosol. ${ }^{20}$ Curtis and coworkers have reported the synthesis of copper organosol using copper acetate and PVP. ${ }^{21}$ Chen and coworkers ${ }^{22}$ have used copper nitrate and a phase transfer reagent for the synthesis of alkanethiolate protected copper clusters. Self-assembled monolayers of alkanethiolate on copper particles using thiols of different chain lengths in ethanol have also been reported. ${ }^{23,24}$ Leung et al. has reported the synthesis of copper nanocrystals using the polypyrrole film by electrochemistry. ${ }^{25}$

A number of peptides have been designed for the stabilization of gold nanoparticles aiming to interact with biomolecules. ${ }^{26}$ This design reflects the usefulness of cysteine as an anchoring group in polypeptide to stabilize metal nanoparticles under different physiological conditions. L-cysteine is a small, zwitterionic molecule, and well used in biochemical and electrochemical research. For example, L-cysteine on gold surface was used to immobilize protein molecules. ${ }^{27-29}$ Understanding the characteristics of cysteine on a solid surface is an important issue in protein study as well as for the detection of amino acid molecules. ${ }^{27-29}$ Mandal et al. have synthesized cysteine functionalized silver nanoparticles in aqueous system. ${ }^{30} \mathrm{Kim}$ et al. have studied the interaction of gold nanoparticles with cysteine. ${ }^{31}$ Burt and coworkers ${ }^{32}$ have shown that gold nanoparticles directly conjugated to globular protein, Bovine serum albumin, via S$\mathrm{Au}$ bonding provides more surface area for interaction with external species. On the other hand, thiol derivatized nanoparticles of noble metals are particularly fascinating in the sense that they can be redispersed in different solvent systems. ${ }^{33}$ Terminally functionalized alkanethiols and arenethiols ${ }^{22,34,35}$ have already been demonstrated for their affinity towards noble metal nanoparticles. Very recently Weckhuysen et al. have reported the synthesis of copper particles in aqueous solution using amino acids. ${ }^{36}$ Though, the research on stabilization of copper nanoparticles has grown up, studies on the interaction of copper nanoparticles with biomolecules are still progressing. Nonetheless, reports on copper nanoparticles are far fewer compared to its gold and silver counterparts, mainly due to its susceptibility towards oxidation. Therefore, synthesis of stable copper particles in organic solvent is in the forefront of nanoparticle research.

Based on the above attributes, we herein report the synthesis and stabilization of copper organosol by a biomolecule L-cysteine and 1-dodecanethiol (DDT) in toluene, 
and characterized the particles to identify $\mathrm{Cu}-\mathrm{S}$ interactions. Copper particles were synthesized in single-phase system with cysteine and alkanethiolate protecting monolayer. Fresh samples exhibit material properties that are very similar to those of the alkanethiolate protected transition metal nanoparticles. For instance the as-produced particles are of spherical shape and exhibit a Mie scattering profile with an optical absorption spectrum. In this synthesis copper stearate was used for the first time as $\mathrm{Cu}$ (II) precursor. The as-synthesized particles are stable for a couple of months and then aggregates slowly. The synthesized particles were characterized by UV visible, TEM, XPS, XRD and FTIR analysis. FTIR study confirmed that the interaction of copper particles with cysteine have been accomplished by a thiolate bond and coordination of the amine group of the cysteine moiety to the copper surface. It was found from the FTIR spectra that carboxylate functionality do not interact with copper surface due to the dielectric environment offered by toluene. It was observed that when copper acetate was used instead of copper stearate, the particles loose their stability. This might be due to the bulky size of the stearate moiety, which provide steric barrier against easy oxidation and particle agglomeration. The particles could be dried under vacuum and could be redispersed in a series of solvents of lower dielectric constant to make the dispersion effective for catalysis. The synthesized particles were found to be an effective catalyst for the synthesis of octylphenyl ether.

\section{Experimental Section}

2.1. Reagents. All the reagents used were of $A R$ grade. Copper acetate and stearic acid was obtained from Merck. Sodium borohydride was purchased from Aldrich. All the solvent used (methanol, toluene and THF) was of AR grade and obtained from Sisco Research Laboratory, India and was distilled before use.

2.2. Instruments. All the absorption spectra were recorded in a Spectrascan UV 2600 spectrophotometer (Chemito, India) and the solutions were taken in a $1 \mathrm{~cm}$ wellstoppered quartz cuvette. The spectra were recorded against a reference solvent in a 
reference cell in the double beam spectrophotometer to subtract solvent background. Transmission Electron Microscopic (TEM) analysis were performed in a Hitachi H-9000 NAR instrument on samples prepared by placing a drop of fresh copper organosol solution on $\mathrm{Cu}$ grids precoated with carbon films, followed by solvent evaporation under vacuum. Fourier Transform Infra Red (FTIR) spectral characteristics of the samples were collected in transmittance mode with Nexus 870 Thermo-Nicolet instrument coupled with a Thermo-Nicolet continuum FTIR microscope. One drop of the test solution was placed on a $\mathrm{KBr}$ pellet and was dried under vacuum for $6 \mathrm{~h}$ before analysis. The FTIR spectrum was recorded over 32 scans of each sample and the background spectrum was automatically subtracted. The XPS analysis was performed using a Fison (VG) ESCA 210 electron spectroscope for chemical analysis. The X-ray diffraction (XRD) pattern was recorded in an $X^{\prime}$ pert pro diffractometer with $\mathrm{Cu}\left(\mathrm{K}_{\alpha}=1.54056\right)$ radiation. The ${ }^{1} \mathrm{HNMR}$ spectrum was recorded in a Bruker AC $200 \mathrm{MHz}$ NMR Spectrometer.

2.3. Synthesis of Copper Stearate. The precursor copper stearate was prepared from copper acetate. Methanolic solution of stearic acid and copper acetate (10:1) was mixed together and the precipitate obtained (insoluble in methanol) was washed thoroughly with methanol in order to make it free from the starting materials copper acetate or stearic acid (both are soluble in methanol). The obtained green precipitate was dried and characterized by powder XRD pattern, TLC and the FTIR analysis to confirm that the sample is free from unreacted stearic acid or copper acetate. Formation of the copper stearate was also confirmed from the melting point of the prepared solid. The details characterization of copper stearate is given in Supporting Information.

2.4. Synthesis of Copper Organosol in Toluene. Synthesis of copper organosol was carried out by exploiting a single-phase procedure using copper stearate as the $\mathrm{Cu}$ (II) precursor. Copper stearate solution was made in tetrahydrofuran (THF) and was used as stock solution $\left(10^{-1} \mathrm{M}\right)$. Then $200 \mu \mathrm{L}$ copper stearate solution was diluted with toluene and final volume of the toluenic solution became $30 \mathrm{~mL}$. Nitrogen gas was purged into the solution to remove the dissolved oxygen. After that $0.005 \mathrm{~g}$ of solid L-cysteine was added to the toluenic mixture. Final concentration of $\mathrm{Cu}(\mathrm{II})$ in toluene was $0.66 \mathrm{mM}$ and 
that of the ligand cysteine was $1 \mathrm{mM}$. Finally the mixture was treated with $2 \mathrm{mg}$ of solid sodium borohydride and was shaken vigorously. With gradual shaking the green color gradually faded down and after $25 \mathrm{~min}$ the solution became colorless indicating the formation of colorless $\mathrm{Cu}(\mathrm{I})$. On further shaking for another $20 \mathrm{~min}$, appearance of light yellow tinge within the solution indicates the onset of evolution of copper nanoparticles in toluene. When DDT was added in lieu of cysteine then the green color of the solution disappeared instantaneously upon the addition of DDT to the toluenic solution of copper stearate and on further shaking for another $20 \mathrm{~min}$, the colorless solution became yellow indicating the formation of copper organosol in toluene. Absorption measurement of the resulting solution functionalized by cysteine shows an absorption band with a new maximum at $487 \mathrm{~nm}$, and the copper organosol protected by DDT have the absorption maximum at $458 \mathrm{~nm}$, which corresponds to the typical plasmon band of copper nanoparticles. The solution was kept for $24 \mathrm{~h}$ so that the excess $\mathrm{NaBH}_{4}$ get decomposed and hence a completely reducing agent free copper organosol in toluene was obtained. The color of the solution persists over a couple of months with unaltered spectral profile.

2.5. Catalysis by Copper Organosol. In a typical set $30 \mathrm{~mL}$ of copper organosol in toluene was prepared in a round bottom flask and then toluene was evaporated using a rotary evaporater. After that, $0.4 \mathrm{mM}$ of iodobenzene and $0.8 \mathrm{mM}$ of $\mathrm{Cs}_{2} \mathrm{CO}_{3}$ were introduced into the flask. The flask was then purged with nitrogen for 20 min and finally $6 \mathrm{~mL}$ of 1-octanol was added to the mixture using a syringe. The mixture was then refluxed under nitrogen gas atmosphere at a temperature of $\sim 120^{\circ} \mathrm{C}$ for $3 \mathrm{~h}$ under continuous stirring condition. The reaction mixture was then filtered through a silica gel packed column and the product was collected in portions, dried under vacuum with gentle heating. The pale yellow colored liquid product was purified by column chromatography (silica gel: petroleum ether/ethylacetate 50:1). The product was characterized from the ${ }^{1} \mathrm{HNMR}$ data and yield of the product was found to be $\sim 91 \%$. 


\section{Results and Discussion}

\subsection{UV-visible Spectra of the Copper Organosol System. Copper stearate}

solution in toluene has an absorption band at $664 \mathrm{~nm}$, which could be attributed to the ligand-to-metal-charge-transfer (LMCT) band ${ }^{37}$ of copper stearate (trace a, Figure 1). When solid cysteine and sodium borohydride was added to it and the solution was shaken, then the green color gradually faded down. After about $25 \mathrm{~min}$, the solution became colorless indicating the formation of colorless $\mathrm{Cu}(\mathrm{I})$ species in toluene. The colorless solution does not show any absorption maxima in the visible region (trace $b$, Figure 1). On further shaking for another $25 \mathrm{~min}$, yellow tinge in the solution sets in indicating the onset of evolution of copper organosol in toluene. Scheme 1 shows the schematic presentation for the copper organosol synthesis functionalized by L-cysteine. On the other hand, when DDT was used in lieu of cysteine it was observed that upon the addition of DDT to the copper stearate solution, the green color disappeared instantaneously, due to the formation of $\mathrm{Cu}(\mathrm{I})$ species. On further shaking for another 20 min persistence yellow color appeared indicating the onset of evolution of copper particles. As cysteine is sparingly soluble in toluene compared to DDT, so it reduces $\mathrm{Cu}(\mathrm{II})$ to $\mathrm{Cu}(0)$ slowly, which was also reflected in the observed particle size. The synthesized copper nanoparticles functionalized with cysteine shows absorption maxima at $487 \mathrm{~nm}$. The optical spectrum of this is illustrated in Figure 1 (trace c). On the other hand, DDT protected copper particle shows a $\lambda_{\max }$ at $458 \mathrm{~nm}$ (trace d, Figure 1). The absorption peaks could be attributed to the excitation of plasmon resonance or interband excitation and are characteristic properties of the metallic nature of the particles. ${ }^{38}$ The observed $\lambda_{\max }$ is quite blue shifted than the reported value for copper nanoparticles. ${ }^{18,20 b, 39}$ Similar result was also observed by Guo et. al. ${ }^{40}$ The lower value of $\lambda_{\max }$ might be due to the special interaction between the ligands (cysteine and DDT) molecule, counter ion stearate and copper nanoparticles. The lower value of $\lambda_{\max }$ could also have a bearing on the smaller particle diameter. ${ }^{36,41}$

3.2. TEM Microanalysis. TEM has been a powerful tool in investigating the nanoparticles molecular dimensions and structures. ${ }^{42-44}$ Figure $2 \mathrm{a}$ shows the 
representative TEM micrograph of copper organosol functionalized by cysteine. From TEM images the average particle size was found to be $11.5 \pm 0.5 \mathrm{~nm}$ (Figure 2a). In addition to these well-dispersed small particles TEM measurement also shows the presence of only a very few larger $(\sim 15.5 \mathrm{~nm})$ particles of spherical shape. It is more likely that the nucleation stage is somehow prolonged over time because of the use of $\mathrm{NaBH}_{4}$ and might be responsible for the wide size distribution. Formation of the larger particles might also be accounted by the sacrificial dissolution of smaller particles. Additional contributions to the formation of these morphologically well-defined nanocrystals might be from the particle aggregates where the molecular mechanism might involve the desorption of ligands leading to the fusion and stacking of the particles. ${ }^{22}$ Figure $2 \mathrm{~b}$ shows the particle size histogram for the cysteine functionalized copper organosol system. From the particle size histogram it is clear that the particles have narrow size distribution. Copper particles protected by dodecanethiol are quite smaller than the cysteine stabilized particles. The average particle size was found to be $8.6 \pm 0.2 \mathrm{~nm}$. It can be seen that, in general the particles are of spherical shape (Figure 2c) and have narrower size distribution.

Cysteine stabilized copper particles are rather larger than the DDT stabilized particles. Between DDT and cysteine, DDT is a stronger reducing agent and hence it reduces the $\mathrm{Cu}$ (II) stearate first to the corresponding $\mathrm{Cu}(\mathrm{I})$ species in presence of $\mathrm{NaBH}_{4}$. Which on further reduction transformed to $\mathrm{Cu}(0)$ stabilized by the ligand DDT. Cysteine is sparingly soluble in toluene and hence it functionalizes the copper surface slowly. Since the rate of reduction is faster for DDT and its capping seems to be facile from the steric consideration, hence it produces smaller particles compared to cysteine-capped particles. This study demonstrates that for the synthesis of copper organosol the stabilizing ligand influences both the particle growth rate and ultimate particle size.

3.3. X-ray Photo Electron Spectroscopy. The direct evidence for the formation of $\mathrm{Cu}(0)$ is obtained from the XPS analysis. Figure 3 shows XPS spectrum of the copper particles. The XPS measurement of the cysteine functionalized copper particles shows two peaks at 932.7 and $952.5 \mathrm{eV}$ corresponding to $\mathrm{Cu} 2 \mathrm{p}_{1 / 2}$ and $\mathrm{Cu} 2 \mathrm{p}_{3 / 2}$ respectively which could be attributed to $\mathrm{Cu}(0) .{ }^{35}$ No peak for the $\mathrm{CuO}$ or $\mathrm{Cu}_{2} \mathrm{O}$ was detected as such. 
This clearly confirms that the $\mathrm{Cu}-\mathrm{S}$ bond formed by the cysteine molecule could form a good barrier layer that protects the copper cores from oxidation.

3.4. XRD Analysis. The $X$-ray diffraction pattern of the synthesized particles functionalized by cysteine moiety is shown in Figure 4 . The diffraction peaks at $2 \theta=$ $43.4^{\circ}, 50.37^{\circ}, 73.99^{\circ}, 89.87^{\circ}$ and $95.68^{\circ}$ are indexed as (111), (200), (220), (311) and (222) planes of fcc of the cubic copper phase. The lattice constant value was calculated to be $3.614 \AA$ which is in good agreement with the literature value. ${ }^{44,45}$ Cupric oxide $(\mathrm{CuO})$ or cuprous oxide $\left(\mathrm{Cu}_{2} \mathrm{O}\right)$ was not detected as such which confirmed that the synthesized particles does not suffer oxidation problem. The observation of diffraction peaks for the copper nanoparticles indicate the crystalline nature of this particle in this size range while its broadening is related to the reduced particle size. ${ }^{46}$ The particle size calculated from the Scherrer formula was $18.2 \mathrm{~nm}$.

3.5. FTIR Analysis. The amino acid cysteine (formula, $\mathrm{H}_{2} \mathrm{~N}-\mathrm{CH}\left(\mathrm{CH}_{2} \mathrm{SH}\right.$ )$\mathrm{COOH}$ ) plays an important role in defining the tertiary structure of proteins through disulfide (cystine) bridges. In our study, free thiol groups in cysteine molecules have been used to bind to colloidal copper and thereby stabilize them. The surface functionalization of copper particles with cysteine was accomplished as described in the Experimental Section. Figure 5 shows the FTIR spectrum recorded from the copper organosol along with the spectrum recorded from cysteine powder. The prominent -SH vibrational band centered at c.a. $2551.31 \mathrm{~cm}^{-1}$ is clearly seen in the free cysteine molecule (Figure 5, trace a) but vanishes upon coordination of these molecules with colloidal copper surface (Figure 5, trace b). This is a strong evidence of surface binding of cysteine molecule with the copper surface via a thiolate linkage. The result agrees well with earlier studies on alkanethiol modification of gold nanoparticles as reported by Mandal ${ }^{30}$ and Murray et al. ${ }^{47}$ The N-H stretch of the cysteine molecule was observed at $3340 \mathrm{~cm}^{-1}$ (Figure 5, trace a). While upon coordination with copper surface the weak peak red shifted to $3440 \mathrm{~cm}^{-1}$ (Figure 5, trace b). The peak position suggests an interaction of the amine group with the copper particles. Further, the carboxylate stretch of the cysteine molecule was observed at $1610 \mathrm{~cm}^{-1}$ (Figure 5, trace a) and that of copper organosol 
system functionalized with cysteine was observed at $1625 \mathrm{~cm}^{-1}$ (Figure 5, trace b). Therefore, the peak slightly red shifted, might be due to the counter ion stearate, which also have carboxylate functionality. In the present case as toluene is used as solvent so we do not anticipate any interaction of the carboxylate group with the copper surface due to the solvent polarity. ${ }^{30}$

FTIR spectroscopy has been used previously to examine the conformational structure of the particle bound organic ligand monolayers, where energies of the symmetric $\left(\mathrm{d}^{+}\right)$and antisymmetric $\left(\mathrm{d}^{-}\right)$stretching vibrations of the methylene units have been taken as a sensitive diagnostic indicator for the ordering of the adsorbed alkanethiolate molecules. ${ }^{48}$ For dodecanethiol molecule these two bands were observed at (d) 2923.55 and $\left(d^{+}\right) 2854.131 \mathrm{~cm}^{-1}$ (Figure 6, trace a) respectively but on coordination with copper particles, these bands slightly blue shifted to (d') 2919.70 and $\left(d^{+}\right) 2850.27$ $\mathrm{cm}^{-1}$ which confirmed the binding of DDT to the copper surface (Figure 6 , trace b). ${ }^{48 \mathrm{~b}}$ Thus, one can see that the copper particle bound dodecanethiolate monolayers resemble the liquid state structure, presumably with a high density of gauche defects. This is consistent with previous observations with other transition metal nanoparticles. ${ }^{48,49}$ The peak for -SH stretching appears at $2555 \mathrm{~cm}^{-1}$ for free DDT molecule (Figure 6, trace a) but vanishes upon coordination with the copper surface (Figure 6, trace b) which confirms the strong binding of copper surface with DDT molecule via a thiolate bond.

3.6. Mechanism of Particle Formation. If methanol is added to the copper organosol system in toluene then the particles get precipitated due to the change in solvent polarity. ${ }^{38 \mathrm{~b}}$ The light yellow colored solid could be easily redispersed in a series of solvents with lower dielectric constant. UV-vis spectroscopy and TEM image indicate the presence of well-dispersed particles even when this solid was redispersed in the desired organic solvent. Formation of this intractable yellow solid after removal of the solvent could be attributed to the cross-linking of the loops and tails of the ligands surrounding the copper particles. The resistance of the solution against particle aggregation and interparticle contact could be explained by cross-linking of the ligand cysteine to the metal surface, possibly catalyzed by the metal. ${ }^{21}$ This would be expected to result in highly effective steric stabilization which could account for the redispersible 
nature of the yellow solid, as well as the inability of the miscible non-solvents to induce interparticle contact. The precise nature of the cross-linking reaction is uncertain, might be due to the coupling action of the ligand cysteine. ${ }^{21}$

Cysteine molecule binds the copper surface via a thiolate bond and an amine linkage, which was confirmed from FTIR spectrum. It has been reported that cysteine molecule could bind via thiolate as well as electrostatically via the carboxylic acid group in an aqueous solution. ${ }^{21,30}$ However, in our case due the dielectric environment offered by the solvent toluene, electrostatic stabilization via the carboxylic acid group is prohibited and hence the stabilization force is mainly due to the thiolate bond and the amine linkage. Synthesized copper particles are therefore stabilized by steric force offered by cysteine molecule as well as by the bulky counterion stearate. Furthermore, the surface layer of covalently bound cysteine molecules could cross-link to the copper particles.

We have considered three different ligands in the system. All of which could serve the purpose of capping agent individually. However, cysteine is the only one over here which can coordinate to the copper surface with more than one donor points forming most stable particles like metal chelates. ${ }^{50}$ On the other hand, monodentate DDT stabilizes the particles because of strong $\mathrm{Cu}-\mathrm{S}$ interaction. However, stearate alone could not impart stability with its oxygen donor point. On the contrary, borohydride react with copper stearate to generate $\mathrm{Cu}(0)$ and extra $\mathrm{BH}_{4}{ }^{-}$act as a nucleophile that shifts the Fermi level potential ${ }^{51}$ to more negative values, thus making the particles more vulnerable to oxidation. In the presence of excess borohydride, the charged particles may release electrons to residual oxygen in the solution, which would fully prevent the formation of surface copper oxide.

We have been interested in the preparation of copper organosol from inexpensive starting materials of low toxicity. In literature there are very few reports on copper organosol synthesis either by harsh synthetic condition, cumbersome reaction routes or using toxic organometallic reagents. ${ }^{18-21}$ Copper stearate is easy to prepare and is soluble in THF. The copper stearate solution in THF could be diluted by toluene where it reacts with $\mathrm{NaBH}_{4}$ to produce the $\mathrm{Cu}(0)$ in the presence of cysteine or DDT. The importance of copper stearate ion is that due to its bulky size it can stabilize the synthesized copper 
particles providing steric barrier to the generated copper particles that is essential to stabilize a nanoparticle system in organic solvents. Rao et al. have reported the solvothermal synthesis of nanocrystals of semiconducting and metal chalcogenides using the stearate precursors of the metals and discussed the importance of stearate ion for the steric stabilization. ${ }^{52}$ When copper acetate was used instead of copper stearate, then the synthesized particles loose their stability and aggregates.

3.7. Particle Aggregation. Under ambient condition, copper particles are stable enough for a couple of months without any morphological change. After that the particle aggregation sets in, might be due to the cross-linking of the ligands. The synthesized particles also might aggregate due to the hydrogen bond formation of the ligands. Very recently we have reported the self-assembly of silver nanoparticles via hydrogen bonding interaction of resorcinol. ${ }^{53}$ The aggregation occurs presumably due to the hydrogen bond formation in case of cysteine only but not for DDT case. On the other hand for DDT functionalized particles hydrogen bonding is not possible but DDT oxidizes to form the disulfides which brings the particles together. ${ }^{35}$ For the latter case the aggregation occurs supposedly by the oxidation of the copper cores and desorption of the ligand leading to the stacking of the particles. Again, the thiols undergo oxidation to form the disulfides, which bring the particles together. ${ }^{54}$ Scheme 2 shows the process of particle aggregation via hydrogen bonding in case of cysteine. The TEM image for cysteine functionalized (Figure 7a) and DDT protected (Figure 7b) copper particles after two months is shown in Figure 8.

3.8. Reaction without Borohydride. If we perform the reaction in absence of sodium borohydride but with excess amount of thiol then it has been observed that DDT itself can reduce as well stabilize the particles and simultaneously oxidizes to disulfide. ${ }^{35}$ Here due to the presence of excess amount of thiol it simultaneously reduces copper stearate to $\mathrm{Cu}(0)$ and the excess thiol present in the system stabilizes the evolved particles. Particles synthesized by DDT are stable for a week only and after that they precipitate down from the solvent. As cysteine is sparingly soluble in toluene it could not 
reduce copper stearate directly in absence of borohydride. It has been reported that the size and shape of copper colloids could be controlled by various additives. ${ }^{46}$ Upon the addition of DDT to the toluenic solution of copper stearate the solution became colorless indicating the formation of colorless $\mathrm{Cu}(\mathrm{I})$ in toluene. After $15 \mathrm{~min}$ of addition of DDT to the copper stearate solution the yellow tint in the toluenic solution indicates the nucleation of $\mathrm{Cu}(0)$. UV-vis spectra were recorded at different time interval. With time the yellow color deepens with successive increase in absorbance value. Initially the absorbance increases with gradual red shifting of the $\lambda_{\max }$, which implies the increase in particle size as well as the particle concentration. After a certain time the $\lambda_{\max }$ was fixed though the absorbance value was still increasing indicating the increase in particle concentration only. From the UV-vis spectra shown in Figure 8 it could be seen that the $\lambda_{\max }$ was initially at $478 \mathrm{~nm}$ but with time it red shifted to $487 \mathrm{~nm}$ and became fixed at this wavelength with gradual increase in absorbance value. The TEM image of the particles has been shown in Figure 9. From the TEM image it has been observed that most of the particles are in the size range between $10-15 \mathrm{~nm}$. There are also very few larger particles with the particle size in the range of 20-25 $\mathrm{nm}$.

It was observed that when lower chain length thiols like 1-octanethiol (C8) or 1decanethiol (C10) were used instead of DDT, then the particle stability decreases with decrease in chain length of the thiols. The stability of metallic nanoparticles in the solution phase is an important issue, since naked metal nanoparticles are unstable in organic solvents, and therefore, some stabilizers are required to prevent the particles from aggregation. Encapsulation of the particle core with an appropriate shell material offers the means of protection from the surrounding environment. Long chains alkanethiols interact with each other via van der Waals forces, and form a compact monolayer that protects the underneath metal from oxidation and aggregation. Although the alkyl chain lengths of the alkanethiols are unable to induce a large variation in the particle morphology but it is very interesting to note the stability of the copper particles in thiols of varying chain lengths. ${ }^{24}$ The TEM images of the copper particles using 1-octanethiol (C8), 1-decanethiol (C10) is given in SI. It was observed that the particle did not differ in size with the decrease in the chain length of the thiols, but the chain lengths of the alkanethiols have a profound influence on the stability of the copper particles. ${ }^{10 \mathrm{a}} \mathrm{The} \mathrm{Cu}-$ 
C10 colloid is stable for 4-5 days but the $\mathrm{Cu}-\mathrm{C} 8$ is stable for 1 day only and after that the particles precipitate down from the solution. Here copper particles are negatively charged due to the adsorption of the stearate ion. Alkanethiols bound to the copper surface via headgroups and are connected to the outer layer through hydrophobic interactions. ${ }^{55}$ Thiols, due to strong thiolate bonding with the copper surface provides the stability to the copper surface. Thus, the steric stabilization arising due to the attachment of the thiols on to the metallic particles causes a screening of the unbalanced attractive van der Waals force. ${ }^{56}$ Therefore, smaller chain lengths thiols cannot provide enough hydrophobicity, enabling them to overcome the van der Waals attractive forces, and this leads to aggregation and oxidation of the particles. On the other hand, longer chain length thiols are compatible enough to render hydrophobicity to the evolved copper particles and help the particles to remain suspended in solution.

3.9. Reaction with Cyanide Ion. The information from the UV-visible spectroscopy, TEM analysis, XPS and the diffraction pattern (XRD) supports the formation of copper organosol in toluene. Moreover, classical knowledge of chemistry was further employed to substantiate the $\mathrm{Cu}(0)$. If the prepared organosol system is allowed to react with aq. $\mathrm{KCN}$ solution it is found that the yellow color disappeared slowly and the solution became colorless due to the formation of colorless $\left[\mathrm{Cu}(\mathrm{CN})_{4}\right]^{3-}$ complex and then the absorption peak due to $\mathrm{Cu}(0)$ vanishes completely. If $\mathrm{KCN}$ is added in stepwise fashion then it was observed that the $\lambda_{\max }$ gradually blue shifted. The blue shift of the $\lambda_{\max }$ corroborates the shift of Fermi levels of the nanoparticles towards more negative potential and thus making them vulnerable to dissolution. ${ }^{39,57}$ However, gradual increase in $\mathrm{KCN}$ concentration produces a colorless solution due to the formation of $\left[\mathrm{Cu}(\mathrm{CN})_{4}\right]^{3-}$ species.

3.10. Catalysis by Copper Particles. The synthesized particles were found to be an effective catalyst for the synthesis of octylphenyl ether. The whole catalytic reaction is presented in Scheme 3. To examine the catalytic action of the as-synthesized copper organosol, the reaction of iodobenzene with 1-octanol under refluxing condition ( $120^{\circ} \mathrm{C}$ ) was chosen. The ${ }^{1} \mathrm{H}-\mathrm{NMR}$ spectrum (in $\mathrm{CDCl}_{3}$ ) of the product shows the 
presence of five aromatic protons in the range of $\delta 6.9-7.2$ as a broad multiplet. The methylene protons $\alpha$ - to the ether group appeared at $\delta 3.9-4.0$ as quartet and the methylene protons $\beta$ - to the ether group appeared at $\delta 1.4$. Other protons present in the $n$ octyl group appeared at the most upfield position, $\delta 1.2$.

The reaction was catalyzed by the two different types of copper particles functionalized with cysteine and DDT respectively. It was found that the yield of the product was comparable ( $91 \%)$ in both the cases. It can be seen from the TEM images that the average particle size are $12 \mathrm{~nm}$ and $9 \mathrm{~nm}$ respectively for the cysteine and DDT functionalized particles. It is well known that the catalytic activity of a particle depends critically on the size and nature of the particles. Goodman and coworkers ${ }^{58}$ have shown that the metal-oxygen interaction can change as a function of the particle size at the nanoscale, which causes interesting size selectivity in a heterogeneously catalyzed

reaction. Very recently Tsukuda et al. have ${ }^{59}$ shown that the smaller particles are more reactive than the larger particles for the oxidation of benzylic alcohols. In this case, it was observed that the rate of the reaction was slightly higher for the $9 \mathrm{~nm}$ particles but it is not significant and the yield was comparable. As the cysteine stabilized copper particles are more stable so we prefer to exploit those particles for catalytic purpose.

\section{Conclusion}

In summary, in this article we have demonstrated that important amino acid cysteine could be easily surface functionalized on the synthesized copper particles. Copper stearate was found to be an important precursor for the copper organosol synthesis leaving aside copper acetate. Organic alkanethiol capping ligands was also used successfully to stabilize copper nanocrystals. The precursor has several important factors: the counter ion stearates sterically stabilizes the particles and therefore impart stability to the copper crystals, it is easy to prepare and cost effective. The route could be easily extended to prepare nanocrystals of other noble metals like $\mathrm{Au}, \mathrm{Ag}$ and $\mathrm{Pt}$. The advantages of the present route are (i) the as-produced material need not to be handled under inert conditions, (ii) inexpensive reagents could be used and (iii) volatile and toxic, organometallic precursors could be avoided. Moreover, the formation of ordered 
nanoparticles in non-aqueous media certainly is an interesting aspect of our approach that also involves the important amino acid cysteine. The particles were found to be an effective catalyst for the synthesis of octylphenyl ether. Since the particles are redispersible in a series of solvents so it could be used as effective catalyst for several organic reactions, which is under investigation.

Acknowledgement. The authors are thankful to CSIR, UGC and DST, New Delhi and Indian Institute of Technology, Kharagpur for financial support.

\section{References}

1. Puntes, V. F.; Krishnan, K. M.; Alivisatos, A. P. Science 2001, 291, 2115.

2. Park, S.; Gorte, R. J.; Vohs, J. M. Appl. Catal. A 2000, 200, 55.

3. Bowker, M.; Madix, R. J. Surf. Sci. 1980, 95, 190.

4. Bowker, M.; Madix, R. J. Surf. Sci. 1982, 116, 549.

5. Dhas, N. A.; Raj, C. P.; Gedanken, A. Chem. Mater. 1998, 10, 1446.

6. Casella, I. G.; Cataldi, T. R. I.; Guerrieri, A.; Desimoni, E. Anal. Chim. Acta 1996, 335, 217.

7. Manbeck, G. F.; Lipman, A. J.; Stockland, R. A.; Freidl, A. L.; Hasler, A. F.; Stone, J. J.; Guzei, I. A. J. Org. Chem. 2005, 70, 244.

8. Wang, H.; Huang, Y.; Tan, Z.; Hu, X. Anal. Chim. Acta A 2004, 526, 13.

9. Nath, S.; Ghosh, S. K.; Panigrahi, S.; Thundat, T.; Pal, T. Langmuir 2004, 20, 7880 .

10. (a) Praharaj, S.; Ghosh, S. K.; Nath, S.; Kundu, S.; Panigrahi, S.; Basu, S.; Pal, T. J. Phys. Chem. B 2005, 109, 13166. (b) Nath, S.; Praharaj, S.; Panigrahi, S.;

Ghosh, S. K.; Kundu, S.; Basu, S.; Pal, T. Langmuir 2005, 21, 10405.

11. Lisiecki, I.; Pilleni, M. P. J. Am. Chem. Soc. 1993, 115, 3887.

12. Ayyappan, S.; Gopalan, R. S.; Subbanna, G. N.; Rao, C. N. R., J. Mater. Res. 1997, 12, 398.

13. Edwards, P. P. Faraday Discuss. Chem. Soc. 1991, 92, 199.

14. Alivisatos, A. P. Science 1996, 271, 933.

15. Henglein, A. Chem. Rev. 1989, 89, 1861.

16. Vossmeyer, T.; Katsikas, L.; Giersig, M.; Popvic, I. G.; Diesner, K.; Chemseddine, A. J. Phys. Chem. 1994, 98, 7665.

17. Johnson, S. R.; Evans, S. D.; Mahon, S. W.; Ulman, A. Langmuir 1997, 13, 51.

18. Lisiecki, I.; Pilleni, M. P. J. Phys. Chem. 1995, 99, 5077.

19. Schmittel, M.; Kalsani, V.; Kienle, L. Chem. Commun. 2004, 1534.

20. (a) Hambrock, J.; Becker, R.; Birkner, A.; Weiß, J.; Fischer, R. A. Chem. Commun. 2002, 68. (b) Bunge, S. D.; Boyle, T. J.; Headley, T. J. Nano Lett. 2003, 3,901 . 
21. Curtis, A. C.; Duff, D. G.; Edwards, P. P.; Jefferson, D. A.; Johnson, B. F. G.; Kirkland, A. I.; Wallace, A. S. Angew. Chem. Int. Ed. Engl. 1988, 27, 1530.

22. Chen, S.; Sommers, J. M. J. Phys. Chem. B 2001, 105, 8816.

23. Sung, M. M.; Sung, K.; Kim, C. G.; Lee, S. S.; Kim, Y. J. Phys. Chem. B 2000 , 104, 2273.

24. Ang, T. P.; Wee, T. S. A.; Chin, W. S. J. Phys. Chem. B 2004, 108, 11001.

25. Sarkar, D. K. ; Zhou, X. J.; Tannous, A.; Leung, K. T. J. Phys. Chem. B 2003, 107, 2879.

26. Levy, R.; Thanh, N. T. K.; Doty, R. C.; Hussain, I.; Nichols, R. J.; Schiffrin, D. J.; Brust, M.; Fernig, D. G. J. Am. Chem. Soc. 2004, 126, 10076.

27. Tengvall, P.; Lestelius, M.; Liedberg, B.; Lundstroem, I. Langmuir 1992, 8, 1236.

28. Sasaki, Y.C.; Yasuda, K.; Suzuki, Y.; Ishibashi, T.; Satoh, I.; Fujiki, Y.; Ishiwata, S. Biophys. J. 1997, 72, 1842.

29. Willner, I.; Katz, E.; Willner, B.; Blonder, R.; Heleg-Shabtai, V.; Bückmann, A. F. Biosens. Bioelectron. 1997, 12, 337.

30. Mandal, S.; Gole, A.; Lala, N.; Gonnade, R.; Ganvir, V.; Sastry, M. Langmuir 2001, 17, 6262 .

31. Aryal, S.; Remant, B. K. C.; Dharamraj, N.; Bhattarai, N.; Kim, C. H.; Kim, H.Y. Spectrochim. Acta A 2006, 63, 160.

32. Burt, J. L.; Gutierrez-Wing, C.; Miki-Yoshida, M.; Jose-Yacaman, M. Langmuir 2004, 20, 11778.

33. Sarathy, K. V.; Kulkarni, G. U.; Rao, C. N. R. Chem. Commun. 1997, 537.

34. Sung, M. M.; Sung, K.; Kim, C. G.; Lee, S. S.; Kim, Y. J. Phys. Chem. B 2000 , 104, 2273.

35. Chen, T. Y.; Chen, S. F.; Sheu, H. S.; Yeh, C. S. J. Phys. Chem. B 2002, 106, 9717.

36. Mesu, J. G.; Beale, A. M.; de Groot, F. M. F.; Weckhuysen, B. M. J. Phys. Chem. B 2006, 110, 17671.

37. (a) Parker, W. L.; Crosby, G. A. J. Phys. Chem. 1989, 93, 5692. (b) Datta, D.; Chakravorty, A. Inorg. Chem. 1983, 22, 1085.

38. Jana, N. R.; Wang, Z. L.; Sau, T. K.; Pal, T. Curr. Sci. 2000, 79, 1367.

39. Salzemann, C.; Lisiecki, I.; Brioude, A.; Urban, J.; Pileni M. P. J. Phys. Chem. B 2004, 108, 13242.

40. Guo, L.; Wu, Z. H.; Ibrahim, K.; Liu, T.; Tao, Y.; Ju, X. The European Physical Journal D 1999, 9, 591.

41. Panigrahi, S.; Kundu, S.; Ghosh, S. K.; Nath, S.; Praharaj, S.; Basu, S.; Pal, T. Polyhedron 2006, 25, 1263.

42. Nikoobakht, B.; Wang, Z. L.; El-Sayed, M. A. J. Phys. Chem. B 2000, 104, 8635.

43. Pan, Z. W.; Dai, Z. R.; Wang, Z. L. Science 2001, $291,1947$.

44. Wang, Z. L. J. Phys. Chem. B 2000, 104, 1153.

45. Feng, J.; Zhang, C. P. J. Colloid Interface Sci. 2006, 293, 414.

46. Filankembo, A.; Pileni, M. P. Appl. Surf. Sci. 2000,164, 260.

47. Templeton, A. C.; Chen, S.; Gross, S. M.; Murray, R. W. Langmuir 1999, 15, 66. 
48. (a) Brust, M.; Walker, M.; Bethell, D.; Schiffrin, D. J.; Whyman, M. J. Chem. Soc. Chem. Comm. 1994, 801. (b) Templeton, A. C.; Wuelfin, W. P.; Murray, R. W. Acc. Chem. Res. 2000, 33, 27.

49. (a) Whetten, R. L.; Shafigullin, M. N.; Khoury, J. T.; Schaaff, T. G.; Vezmar, I.; Alvarez, M. M.; Wilkinson, A. Acc. Chem. Res. 1999, 32, 397. (b) Chen, S.; Huang, K.; Stearns, J. A. Chem. Mater. 2000, 12, 540.

50. Kundu, S.; Pal, A.; Ghosh, S. K.; Nath, S.; Panigrahi, S.; Praharaj, S.; Pal, T. Inorg. Chem. 2004, 43, 5489.

51. Pal, T.; Sau, T. K.; Jana, N. R. Langmuir 1997, 13, 1481.

52. Gautam, U. K.; Seshadri, S. R.; Fiona, M. M.; Peter, M. Chem. Commun. 2001, 629.

53. Panigrahi, S.; Praharaj, S.; Basu, S.; Ghosh, S. K.; Jana, S.; Pande, S.; Vo-Dinh, T.; Jiang, H. J.; Pal, T. J. Phys. Chem. B 2006, 110, 13436.

54. Schedel-Niedrig, T.; Neisius, T.; Böttger, I.; Kitzelmann, E.; Weinberg, G.;

Demuth, D.; Schlögl, R. Phys. Chem. Chem. Phys. 2000, 2, 2407.

55. Nikoobakht, B.; El-Sayed, M. A. Langmuir 2001, 17, 6368.

56. Manna, L.; Scher, E. C.; Alivisatos, A. P. J. Am. Chem. Soc. 2000, 122, 12700.

57. Jana, N.R.; Sau, T. K.; Pal, T. J. Phys. Chem. B 1999, 103, 115.

58. Valden, M.; Lai, X.; Goodman, D. W. Science 1998, 281, 1647.

59. Tsunoyama, H.; Sakurai, H.; Negishi, Y.; Tsukuda, T. J. Am. Chem. Soc. 2005, $127,9374$. 


\section{Figure Captions and Schemes}

Figure 1: UV-vis spectra of (a) copper stearate in toluene (b) $\mathrm{Cu}(\mathrm{I})$ species in toluene (c) copper particles functionalized by cysteine (d) copper particles protected by dodecanethiol in toluene

Figure 2: TEM images of (a) copper particles functionalized by cysteine (b) particle size histograms for the copper particles functionalized by cysteine (c) copper particles protected by dodecanethiol

Figure 3: XPS spectra of copper particles functionalized by cysteine

Figure 4: X-ray diffraction pattern of the copper particles functionalized by cysteine

Figure 5: FTIR spectra of (a) cysteine (b) copper particles functionalized with cysteine

Figure 6: FTIR spectra of (a) dodecanethiol (b) copper particles capped by dodecanethiol

Figure 7: TEM image of the aggregated copper particles from (a) cysteine

(b) dodecanethiol

Figure 8: UV-vis spectra of copper organosol with DDT only

Figure 9: TEM images of copper organosol prepared by DDT only

Scheme 1. Schematic presentation for the copper organosol synthesis functionalized by L-cysteine

Scheme 2. Schematic presentation of the particle aggregation via hydrogen bonding

Scheme 3. Schematic presentation of the catalytic reaction 
Figure 1 


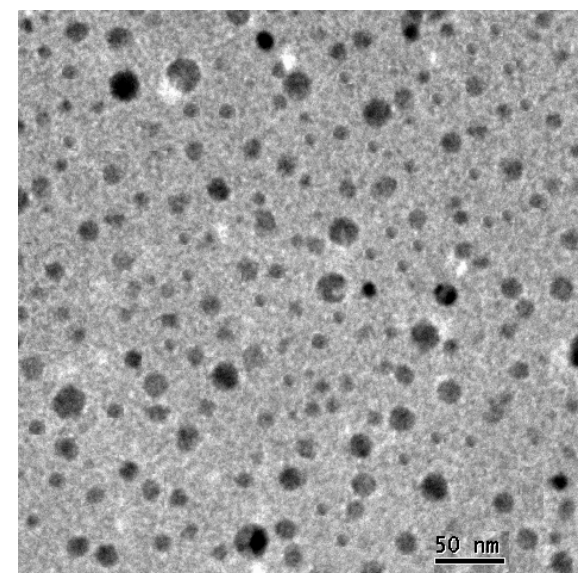

Figure 2a

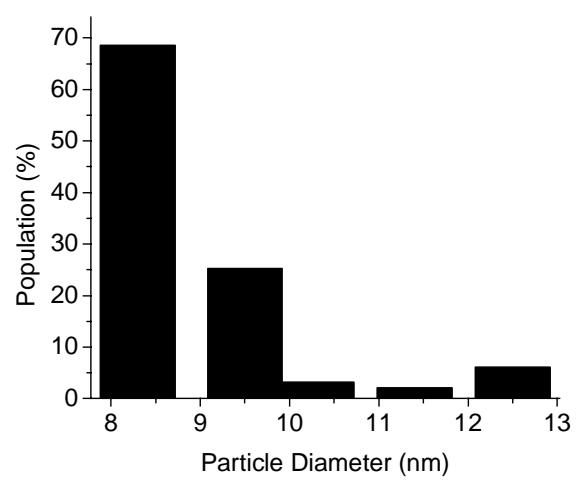

Figure 2b

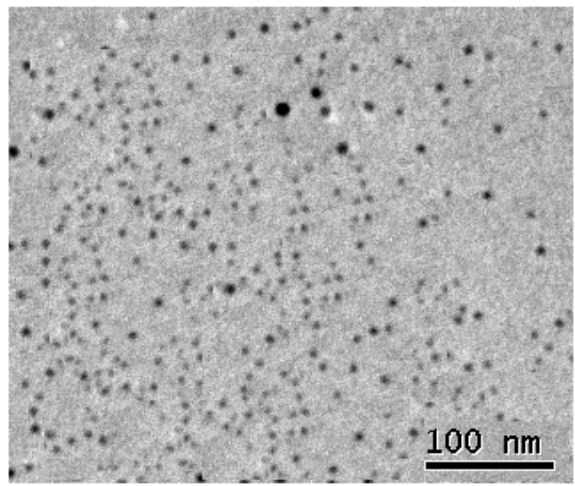

Figure 2c 


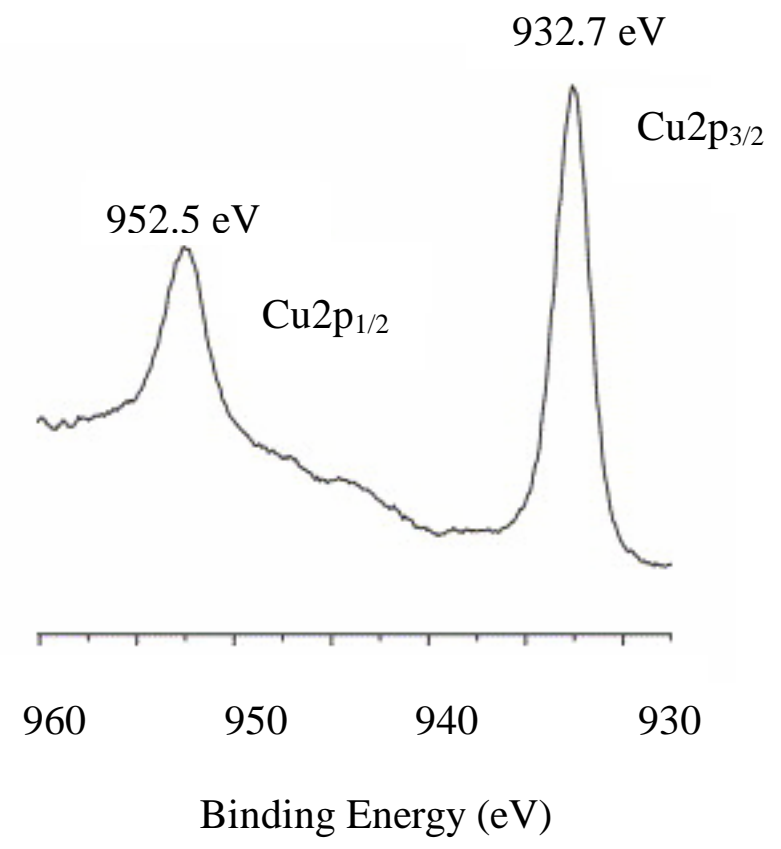

Figure 3 


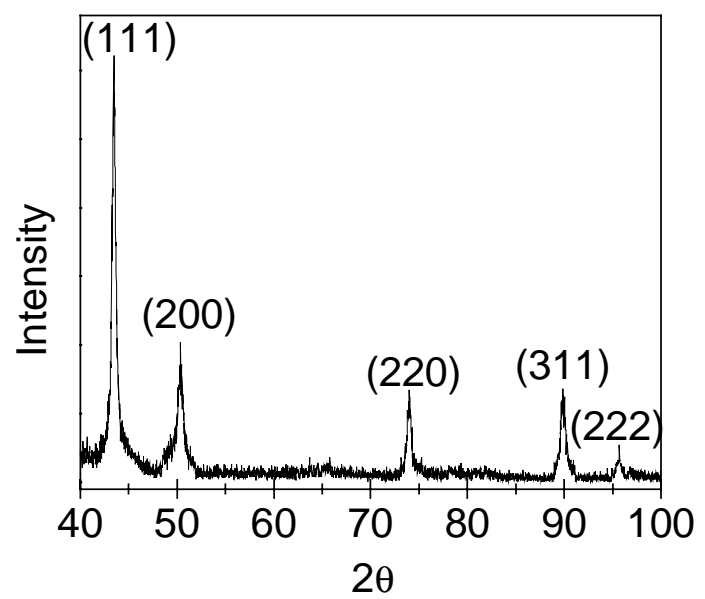

Figure 4 


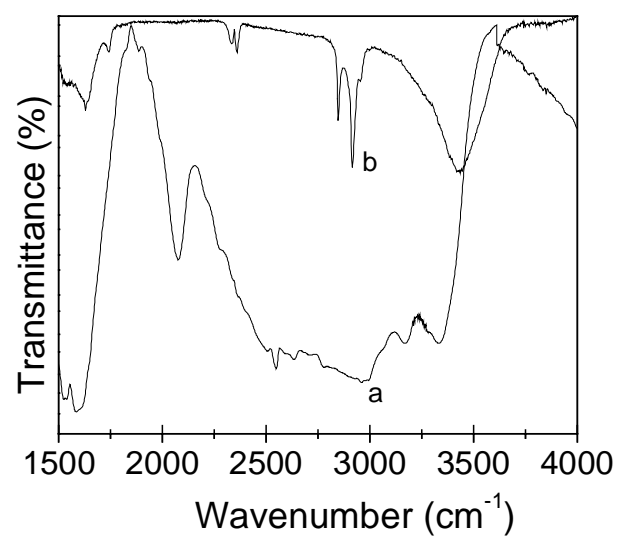

Figure 5 


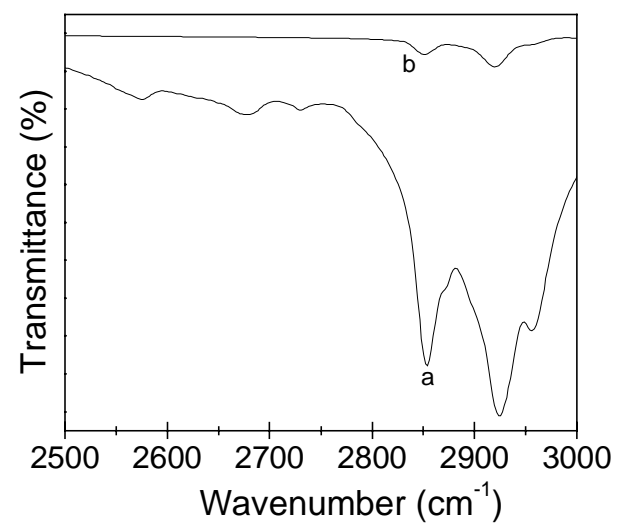

Figure 6 


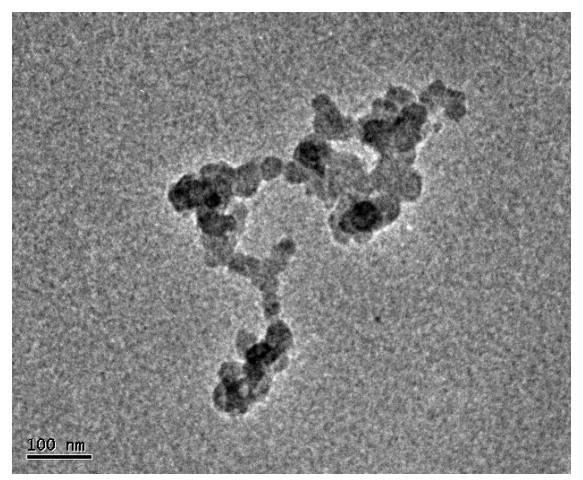

Figure 7a

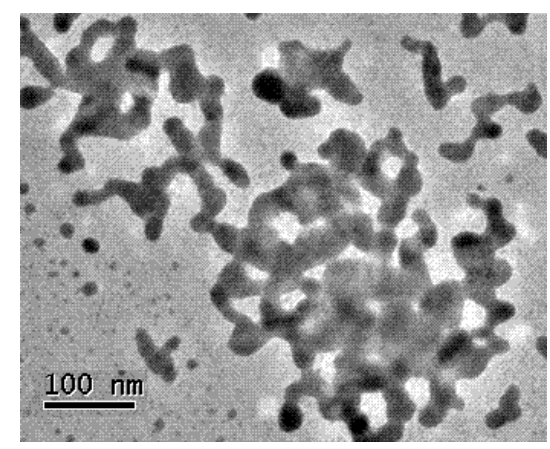

Figure 7b 


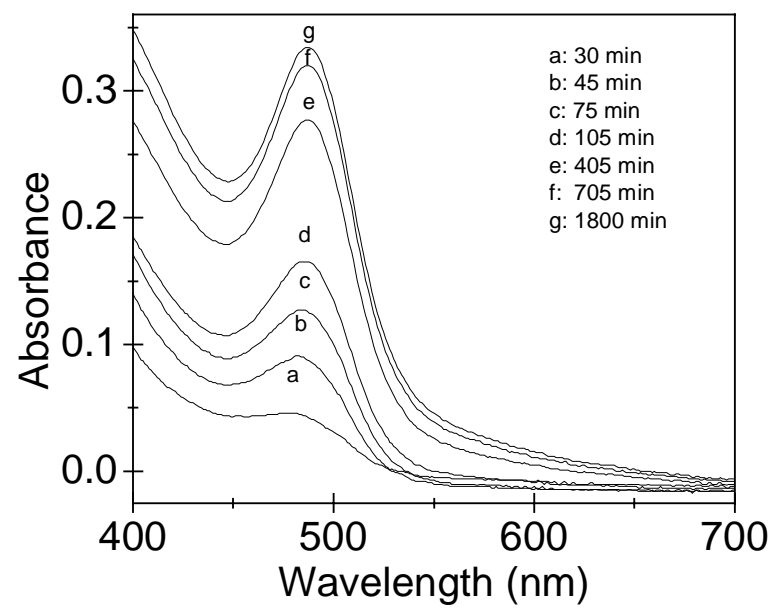

Figure 8 


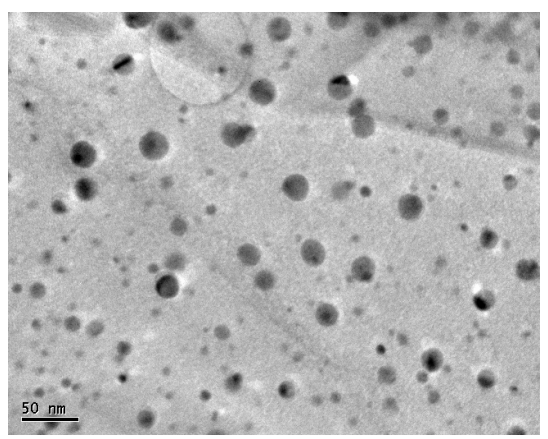

Figure 9 


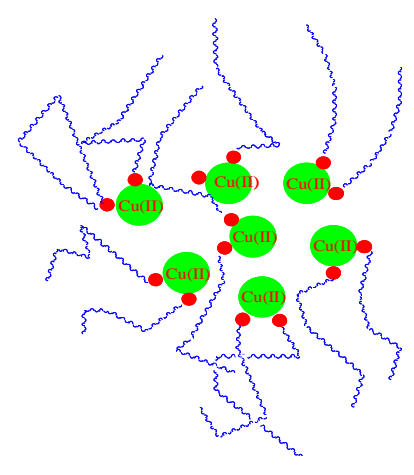

Copper stearate

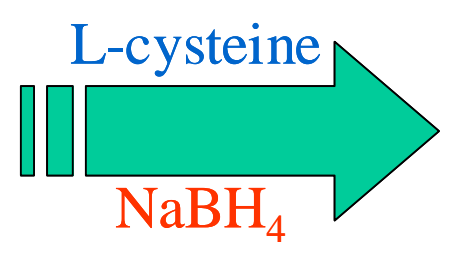

Copper particles functionalized by L-cysteine

Scheme 1 


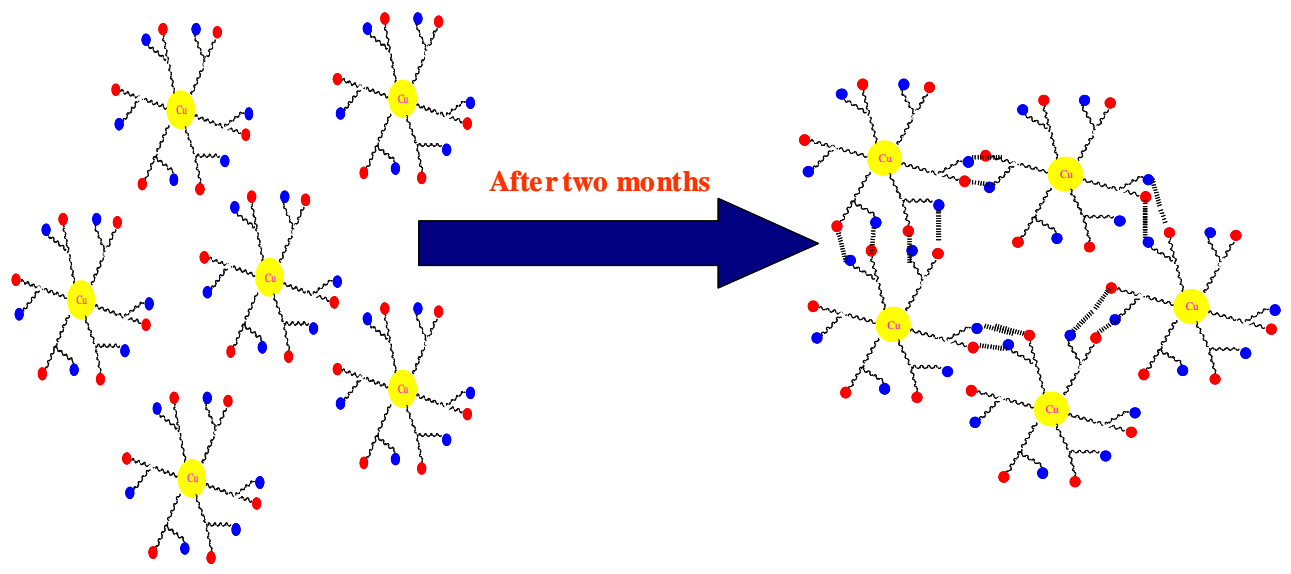

Copper particles functionalized by L-cysteine

Aggregates of copper particles

Scheme 2 


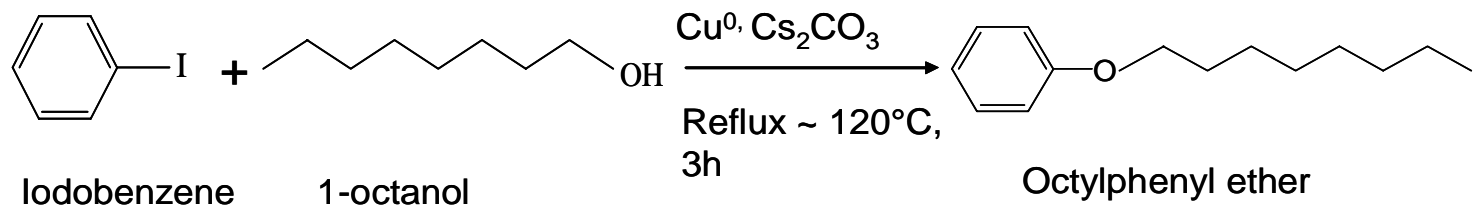

Scheme 3 